\title{
El transnacionalismo político de las asociaciones de inmigrantes en Barcelona
}

\author{
Mihaela Vancea y Àlex Boso \\ Universitat Pompeu Fabra de Barcelona, Españal \\ Universidad de la Frontera, Chile
}

\section{Resumen}

El objetivo de este artículo es explorar en qué medida las asociaciones de inmigrantes se implican en el transnacionalismo político y qué determina las diferencias en su implicación. El estudio compara los niveles de transnacionalismo político de 24 asociaciones de inmigrantes en Barcelona, indagando cómo influyen variables de nivel meso y macro en dicho proceso. Los resultados señalan que el compromiso político transnacional no sigue un patrón generalizado entre las asociaciones de inmigrantes y presenta un nivel relativamente bajo de regularidad. El análisis de los datos también apunta la importancia de la estructura de oportunidades políticas en el país de origen, las redes sociales y la financiación de la organización como elementos clave para entender su nivel de implicación política transnacional.

Palabras clave: Transnacionalismo político, asociaciones de inmigrantes, participación política, estructura de oportunidades políticas, redes sociales.

\section{Abstract}

The political transnationalism of the immigrant associations in Barcelona

This article aims to addresses two fundamental questions: to what extent immigrant associations engage in political transnationalism; and what are the main determinants of their transnational political activism. The study compares the degree of political transnationalism of 24 immigrant associations in Barcelona, by exploring the influence of meso- and macro- level determinants. The results show that transnational political engagement is not generalized among all immigrant associations and presents a relatively low level of regularity. They also indicate the importance of the political opportunity structure in the home country, the social networks and the sources of funds as significant factors to understand the level of their transnational political engagement.

Key words: Political transnationalism, immigrant associations, political participation, political opportunity structure, social networks. 


\section{INTRODUCCIÓN}

7 radicionalmente, la literatura sobre el transnacionalismo migrante se ha centrado en la investigación de las prácticas individuales, considerando al individuo como la principal unidad de análisis (Portes, Guarnizo y Landolt, 1999). Sin embargo, en la última década, son diversos los estudios que han empezado a enfatizar el papel de las asociaciones de inmigrantes y de las redes organizacionales en la definición del transnacionalismo migrante (Faist, 2000a, Portes, 2001, Portes, 2003; Vertovec, 2003), o en desarrollo de procesos que estructuran las prácticas políticas transnacionales (Portes et al., 1999; Itzigsohn, 2000; Østergaard-Nielsen, 2003c).

Este artículo intenta analizar de manera sistemática la involucración de las asociaciones de inmigrantes de Barcelona en actividades y prácticas políticas a través de las fronteras. Gran parte de los estudios realizados en el ámbito europeo señalan la relevancia del contexto de destino (nacional y sub-nacional) en la producción de oportunidades políticas para el desarrollo de las prácticas transnacionales (Bauböck, 2003; Bloemraad, 2005; Martiniello y Lafleur, 2008; Morales y Jorba, 2010).

La perspectiva metodológica abordada en este estudio difiere de este cuerpo de investigación, al estudiar la estructura de oportunidades políticas principalmente en el contexto de origen. Esta línea de investigación nos permite indagar cómo la estructura de oportunidades políticas del contexto de origen puede promover o desalentar el desarrollo de las prácticas transnacionales. De este modo, se pretende cuestionar ciertos análisis convencionales que se centran exclusivamente en la asimilación de los inmigrantes a la sociedad de destino, descuidando los lazos de los migrantes con los países de origen que podrían dar lugar a nuevas formas de acomodación de los migrantes a un contexto más global e interconectado.

La investigación se centra en el estudio comparado de 24 asociaciones de inmigrantes de diferente origen étnico/cultural en la ciudad de Barcelona. Los grandes conglomerados urbanos suelen ser las zonas donde más inmigrantes llegan, principalmente debido a la presencia de mayores oportunidades de trabajo. Barcelona es, de hecho, la segunda zona metropolitana de España y una de las principales zonas de concentración de inmigrantes. Por otro lado, los sistemas políticos de las ciudades suelen ser más abiertos a las afiliaciones transnacionales que los estados-nación (Bauböck, 2003b). 
Históricamente, Barcelona ha sido una ciudad de inmigración. En el siglo XIX y principios del siglo XX, hubo una migración interna hacia Barcelona de otras provincias catalanas y también de varias regiones vecinas debido a una importante mejora en la infraestructura. Con los años 80 y 90, los inmigrantes comenzaron a llegar a Barcelona desde fuera de las fronteras españolas (Solé, 2000). Entre 2000 y 2006, el valor absoluto de las personas registradas con otra nacionalidad que el español (cómo un inmigrante suele ser definido por las estadísticas oficiales que analizan la migración internacional) ha aumentado significativamente en la ciudad de Barcelona. Ésta es la ciudad más importante de la jerarquía del sistema urbano del Área Metropolitano de Barcelona (AMB), donde se concentran la mayoría de las poblaciones de inmigrantes europeos, americanos y asiáticos (Cladera y Elordui-Zapaterietxe, 2004).

El presente artículo plantea dos cuestiones fundamentales en el campo de estudios migratorios. ¿En qué medida las asociaciones de inmigrantes participan en el transnacionalismo político? ¿Cuáles son los principales determinantes del activismo político transnacional que realizan las asociaciones de inmigrantes?

En este artículo se procede de la siguiente manera: en la primera sección, se define el transnacionalismo político de los inmigrantes y se explicita la operacionalización de los conceptos analizados; en la segunda sección, se describen las técnicas de investigación empleados; y en la tercera sección, se explicitan los resultados obtenidos, delimitando las diversas formas de activismo político transnacional desarrolladas por las asociaciones de inmigrantes en Barcelona. Finalmente, se presentan las conclusiones del estudio y se enfatizan futuras líneas de investigación en el tema de interés.

\section{CONCEPTUALIZACIÓN DEL TRANSNACIONALISMO POLíTICO}

El transnacionalismo migrante representa un fenómeno y un concepto relativamente nuevo en el ámbito de las ciencias sociales. Aunque algunas formas de transnacionalismo se han dado desde la primeras experiencias migratorias internacionales, como son los casos de los migrantes repatriados, los inmigrantes que cíclicamente regresan a sus países de origen, las "diásporas comerciales"21 o las "diásporas políticas", ${ }^{22}$ éstas formas de

\footnotetext{
${ }^{21}$ Comunidades compuestas por mercaderes ambulantes que se establecen en jurisdicciones extranjeras con el propósito de dedicarse a las actividades comerciales (Curtin, 1984).

${ }^{22}$ Comunidades forzadas a establecerse en diferentes países como los judíos rusos que escapaban de las persecuciones zaristas en el siglo XX, los armenios para escapar de la opresión turca o la gran diáspora española después de la victoria fascista de ese país (Rischin, 1962; Howe, 1976; Noiriel, 1995; Weil, 1991; Solé, 1995).
} 
relación a ambos lados de la frontera no alcanzaron ni la regularidad, ni la naturaleza rutinaria y crítica de las prácticas y movimientos actuales (Cohen, 1997; Portes et al., 2003; Moctezuma, 2004, 2008; Vertovec, 2009).

La novedad más relevante en la naturaleza del transnacionalismo migrante contemporáneo radica en el creciente número de personas involucradas, la alta intensidad y la regularidad de los intercambios facilitados por los avances tecnológicos, la multiplicidad de actividades que trascienden las fronteras estatales y que requieren este tipo de movimiento geográfico para su cumplimiento y, finalmente, el alto grado de institucionalización de las nuevas prácticas (Itzigshon et al., 1999; Itzigshon, 2000; Portes et al., 2003). Las diferencias conceptuales entre las prácticas transnacionales de nuevo cuño y las clásicas redes migratorias representan la contribución académica más relevante del enfoque transnacional en el desarrollo teórico de los estudios migratorios (Vertovec, 2004).

Basch et al. (1994) en una de las primeras aproximaciones teóricas al trasnacionalismo, definieron este concepto como un amplio abanico de actividades y procesos a través de los cuales los inmigrantes desarrollan y mantienen relaciones sociales diversas que conectan las sociedades de origen y de destino. Otros autores trataron de acotar esta definición al proponer la reducción del concepto de transnacionalidad a aquellas prácticas organizadas por actores non-institucionales a través de las fronteras y que se desarrollan fuera del ámbito de regulación y control estatal (Portes et al., 1999; Portes, 2001; Guarnizo, Portes y Haller, 2003).

Diversos autores han tratado de defender el desarrollo del transnacionalismo migrante como una nueva mirada teórica de la migración, $\mathrm{y}$, consecuentemente, han formulado tipologías relativas a las formas, niveles y grados del transnacionalismo. Smith y Guarnizo (1998) han distinguido entre el transnacionalismo desde arriba (actividades transfronterizas de los gobiernos y corporaciones multinacionales) y el transnacionalismo desde abajo (iniciativas desarrolladas por los actores de la sociedad civil, como las organizaciones no gubernamentales, movimientos sociales, y inmigrantes, que actúan a través de las fronteras estatales).

Portes y sus colaboradores (Guarnizo et al., 2003; Portes et al., 2007) han distinguido entre el transnacionalismo en un sentido "amplio" - un conjunto de prácticas que incluyen actividades (económicas, políticas y socio-culturales) tanto regulares, como ocasionales, y el transnacionalismo "estricto"- concepto que sólo incluye las prácticas regulares. En el mismo sentido, Itzigsohn et al. (1999) y Østergaard-Nielsen (2003) distinguen entre un transnacionalismo político "estrecho" — para definir las activida- 
des y prácticas institucionalizadas y regulares, como son la pertenencia a partidos o asociaciones en el país de origen, y el transnacionalismo político "amplio" - para definir la participación en actividades ocasionales, reuniones o eventos que vinculan políticamente a los inmigrantes con sus lugares de origen.

Østergaard-Nielsen $(2001,2003 b)$ define el transnacionalismo político de los migrantes como diversas formas de participación transfronteriza de los migrantes y los refugiados en la política de su país de origen, ya sean estas formas directas o indirectas (a través de las instituciones del país de acogida o de organizaciones internacionales). La autora identifica cuatro tipos de prácticas y actividades políticas transnacionales llevadas a cabo por los migrantes, a saber: i) política del país de origen (homeland politics); ii) política de la diáspora (diaspora politics); iii) política de inmigración (immigrant politics); y iv) política trans-local (trans-local politics).

Recientemente, algunos autores han destacado el papel de los actores civiles y de los migrantes en el análisis del transnacionalismo, argumentando la importancia de focalizar en las prácticas transnacionales como una noción empírica más operativa (Martiniello y Lafleur, 2008). Los actores civiles y, en particular, las asociaciones de inmigrantes, han tomado un rol privilegiado en el análisis de las practicas transnacionales, principalmente, las cívicas y políticas (Orozco, 2003a; Moctezuma, 2004; Portes et al., 2007; Östergaard-Nielsen, 2001, 2003b; Cordero-Guzmán, 2005; Luque, 2007; Morales y Jorba, 2010).

En este articulo, tomamos como punto de partida la definición del transnacionalismo político de Östergaard-Nielsen (2001, 2003b), adaptada al caso de las asociaciones de inmigrantes. Se ha definido el transnacionalismo político migrante como la participación política transfronteriza (electoral y no electoral) a través de la cual los migrantes tratan de ejercer presión sobre al menos dos comunidades políticas (países de origen y de destino). Dicha participación puede manifestarse directamente a través del voto o la afiliación a un partido político, pero también puede ejercerse indirectamente a través de las instituciones políticas del país de origen (el gobierno, la embajada o el consulado) $u$ otras organizaciones internacionales.

Se ha discriminado entre tres tipos de participación política transnacional: i) política del país de origen; ii) política de inmigración; y iii) política trans-local (Østergaard-Nielsen, 2001, Østergaard-Nielsen, 2003b). También se ha distinguido entre transnacionalismo político migrante "amplio" (cuando este es institucionalizado y sus actividades tienen cierta regularidad) y "estrecho" (cuando los vínculos o prácticas son ocasionales) (Itzigsohn et al., 1999; Østergaard-Nielsen, 2003b; Vertovec, 2009). 
En la política del país de origen se han incluido aquellas actividades y prácticas políticas llevadas a cabo por las asociaciones de inmigrantes y relacionadas con la política interior o exterior del país de origen (voto, participación en campañas electorales, contribuciones monetarias a partidos o candidatos, formas de protesta, acciones informativas sobre eventos políticos sucedidos en el país de origen, etc.).

La política de inmigración se ha definido por aquellas actividades y prácticas políticas que las asociaciones de inmigrantes desarrollan para mejorar la situación legal y socioeconómica de sus connacionales en el país de destino, y que están apoyadas generalmente por el país de origen u otros organismos internacionales (voto o apoyo a un partido político que favorece la ampliación de los derechos de la población inmigrada, formas de protesta en defensa de los derechos de los inmigrantes, acciones informativas sobre dichas cuestiones, manifestaciones social-culturales como formas de incidencia política en el país de destino).

La política trans-local se ha operacionalizado como aquellas actividades y prácticas políticas transnacionales que buscan mejorar la situación de las comunidades locales de origen (pertenencia a una asociación o comité cívico en el país de destino para apoyar a las comunidades de origen, contribuciones monetarias a proyectos comunitarios o a organizaciones de caridad, acciones informativas sobre asuntos socio-económicos y políticos de las comunidades locales de origen, manifestaciones social-culturales como formas de incidencia política en el país de origen, etc.).

De acuerdo con los estudios de movimientos sociales (Koopmans, 1999; Koopmans et al., 2005; Tarrow, 2004) y la literatura académica en el campo de la migración transnacional (Portes, 1999; Basch et al., 1994; Itzigshon, 2000; Portes et al., 2003; Smith, 2003; Portes et al., 2007; Levitt y de la Dehensa, 2003), el concepto de estructura de oportunidades políticas en el país de origen se ha definido en función de tres dimensiones: i) el nivel de libertad (derechos políticos y libertades civiles) en el país de origen; ${ }^{23}$ ii) los derechos políticos formales para los ciudadanos residentes en el extranjero (doble ciudadanía y los derechos de voto externos) $;{ }^{24}$ y iii)

\footnotetext{
${ }^{23}$ La dimensión del nivel de libertades en el país de origen se ha definido en términos de cuan libre (democrático) es un país en términos de derechos políticos y libertades civiles (no libre, parcialmente libre y libre). Hemos utilizado los datos de la Freedom House Annual Global Survey of Political Rights and Civil Liberties, Freedom in the World 2010. (http://www.freedomhouse.org). ${ }^{24}$ La dimensión de los derechos políticos formales para los ciudadanos residentes en el extranjero se ha definido a través de dos indicadores: derechos de voto en el extranjero (sin derecho de voto; derecho de voto con algunas restricciones; con derecho de voto; y derechos de ciudadanía doble (no tienen derecho; tienen derecho con algunas excepciones; derecho pleno). Se han utilizado datos procedentes del proyecto Election Process Information Collection Project (EPIC), (http://www.developmentgateway.com.au/cms/op/preview/sectors/governance/page2812.html);
} 
otras políticas estatales dirigidas a los emigrantes que viven en el extranjero. $^{25}$

El nivel de desarrollo socio-económico en el país de origen se ha operacionalizado a través del Índice de Desarrollo Humano (IDH). Entre las características organizativas destacan las redes sociales y el tipo de asociación (cívica, social, cultural, de la comunidad de origen). ${ }^{26}$ Finalmente, se han recolectado datos acerca de otras dimensiones organizacionales que podían afectar al transnacionalismo político de las asociaciones de inmigrantes analizadas, como el año de la fundación de la asociación, el número de miembros o las fuentes de financiación.

\section{Supuestos TEÓRICOS, DATOS Y SELECCIÓN DE PARTICIPANTES}

Este artículo pretende explorar diversos supuestos teóricos manejados en el campo de estudio del transnacionalismo político migrante. En primer lugar, pretende analizar si la implicación política transnacional de las asociaciones de inmigrantes en Barcelona es o no heterogénea. En segundo lugar, el estudio tratará de observar si cómo apunta la literatura especializada, las asociaciones de inmigrantes con una mayor estructura de oportunidades políticas en el país de origen participan en mayor medida en actividades y prácticas políticas transnacionales. Otro supuesto teórico que será explorado en el estudio es si un menor nivel de desarrollo socio-económico en el país de origen motiva una mayor participación de las asociaciones de inmigrantes en el transnacionalismo político. Por último, se indaga si las características organizativas de las asociaciones de inmigrantes pueden influir en su participación política a través de las fronteras.

La información recogida para este estudio se ha basado principalmente en entrevistas estructuradas (en español e inglés) a representantes/dirigentes de 24 asociaciones de inmigrantes, de diferentes orígenes culturales, en Barcelona. También se han incorporado datos cualitativos procedentes de conversaciones informales con los distintos miembros de las asociaciones de inmigrantes seleccionados y de observaciones estructuradas de reuniones y asambleas de asociaciones. Todas las entrevistas fueron grabadas y

así como otros procedentes de Interparliamentary Union's internet website (http://www.ipu.org/ english/home.htm).

${ }^{25}$ Se ha definido a través de cuatro indicadores (Levitt y de la Dehensa 2003): i) reformas ministeriales y consulares (de naturaleza burocrática); ii) políticas de inversión que tratan de atraer o canalizar las remesas de los migrantes; iii) presencia y protección de servicios a los ciudadanos residentes en el exterior que van más allá de los servicios consulares tradicionales; y iv) políticas simbólicas diseñadas para reforzar el sentimiento de pertenencia y lealtad al país de origen.

${ }^{26}$ Las asociaciones de inmigrantes que se definen como organizaciones políticas son más bien una minoría (Portes et al., 2007). 
transcritas literalmente. Los principales temas o cuestiones abordadas en las entrevistas fueron: origen, tipo y estructura de la asociación; tipo de actividades y prácticas transnacionales (política del país de origen, política de inmigración, política trans-local); e intensidad de las prácticas políticas transnacionales.

Para evaluar si las asociaciones de inmigrantes realmente representan a la ciudadanía transnacional, precisábamos de una muestra representativa de este segmento de población. Sin embargo, una muestra representativa estándar podría obviar aquellas asociaciones transnacionales de inmigrantes que resultan más interesantes: las que se dedican a actividades muy infrecuentes pero importantes, tales como proporcionar grandes donaciones a asociaciones $u$ organizaciones del país de origen, prestar servicios en juntas directivas del país de origen, o participar en protestas y denunciar violaciones de derechos humanos en el país de origen (Verba et al., 1995: 6).

En consecuencia, se tuvieron en cuenta aquellas asociaciones de inmigrantes que correspondían a los grupos culturales políticamente más relevantes en la ciudad de Barcelona, como los argentinos, uruguayos y cameruneses, que la mayoría emigraron de sus países en los años 70 y 80 por razones políticas. También se incluyeron en la muestra aquellas asociaciones que correspondían a los grupos de inmigrantes numéricamente más significativos en Barcelona, en el año de la selección de la muestra: los ecuatorianos, los peruanos, los marroquíes y los paquistaníes.

Se ha aplicado también un cuestionario sobre las políticas gubernamentales dirigidas a emigrantes como reformas burocráticas, políticas de inversión, derechos políticos, servicios del Estado en el extranjero o de prestación de servicios, o políticas simbólicas para reforzar el sentido de pertenencia nacional. El cuestionario se envió por correo postal, correo electrónico y fax a las embajadas y los consulados de los países representados en la muestra de asociaciones. Las embajadas (o consulados) correspondieron a los once países de origen de las asociaciones participantes: Panamá, Argentina, Uruguay, Perú, Ecuador, Marruecos, Camerún, Nigeria, Guinea Ecuatorial, Pakistán y Senegal. También se han incluido datos agregados sobre el contexto socio-económico y político del país de origen.

La selección de asociaciones participantes en esta investigación fue mediante una muestra "intencional". Se seleccionaron las observaciones que iban a ser incluidas en el estudio asegurando la variación de dos factores clave para explicar los diferentes niveles de transnacionalismo político, como son la estructura de oportunidades políticas y el nivel de desarrollo socio-económico en el país de origen, sin tener en cuenta los valores de la 
variable dependiente (King et al., 1994: 137-140). Se desarrolló una tipología específica de las características del país de origen mediante el uso de indicadores compuestos sobre el nivel de desarrollo socio-económico (IDH) y la estructura de oportunidad política (POS) en el país de origen. La variación en el tipo de asociación de inmigrantes (cívica, social, cultural, de la comunidad de origen) fue también tomada en cuenta en la construcción de la muestra. Finalmente, se seleccionaron 41 asociaciones de inmigrantes de 11 orígenes diferentes. Finalmente, 24 fueron las asociaciones de inmigrantes participantes en el estudio. ${ }^{27}$

\section{Delimitando el aCtivismo POLÍtico TRANSNACIONAL}

¿Existen asociaciones de inmigrantes políticamente transnacionales en Barcelona? ¿Qué formas, alcance e intensidad caracterizan su transnacionalismo político? Los resultados de esta investigación sugieren que las asociaciones de inmigrantes practican en grado diverso el transnacionalismo político. De hecho, las actividades políticas ocasionales a través de las fronteras estatales tienden a ser el patrón predominante.

La mayoría de los representantes de las asociaciones entrevistados (91 por ciento) manifestaron que las entidades a las que pertenecen practicaban alguna forma de transnacionalismo político, principalmente actividades relacionadas con la política del país de origen y la política transnacional. También declararon que se implicaban en actividades especificas de la política de inmigración pero, por lo general, sin ningún tipo de ayuda o apoyo del gobierno del país de origen u de otros organismos internacionales. Sólo dos de las 24 asociaciones de inmigrantes participantes en el estudio manifestaron que no estaban involucradas en ninguna forma de transnacionalismo político (Tabla 1).

\footnotetext{
${ }^{27}$ Aunque la muestra comprendía inicialmente 41 asociaciones de inmigrantes no todas fueron finalmente estudiadas. En España las asociaciones de inmigrantes no están obligadas a firmar en el registro cuando deciden poner fin a su actividad, hecho que comportó que un número de asociaciones que constaban en las listas oficiales no existieran de facto en el momento de realizar el trabajo de campo. Algunas asociaciones habían cambiado de dirección, número de teléfono o correo electrónico sin anunciarlo, hecho que dificultó su localización. Una asociación se negó a participar. Éste nivel de respuesta puede considerarse aceptable dado que la mayoría de las asociaciones de la muestra que no respondieron (11) ya no existían en el momento de la recogida de datos (véase Montero et al. 2006; Morales y Jorbá, 2010). La tasa de respuesta del 59 por ciento, no sólo representa más de la mitad de las asociaciones incluidas en la muestra inicial sino que, en verdad, equivale a más de dos tercios de las asociaciones seleccionadas realmente existentes. Además, se ha logrado mantener la representatividad estructural de la muestra, pues el estudio dispone de al menos un caso para cada categoría de las variables de selección.
} 
Tabla 1: Asociaciones de inmigrantes y formas de transnacionalismo político

\begin{tabular}{|c|c|c|c|c|c|c|}
\hline Asociación de inmigrantes & Tipo & $\begin{array}{r}\text { Año } \\
\text { fundación }\end{array}$ & $\begin{array}{r}\text { No. } \\
\text { miembros }\end{array}$ & $\begin{array}{r}\text { Política } \\
\text { país origen }\end{array}$ & $\begin{array}{r}\text { Política } \\
\text { inmigración } \\
\end{array}$ & $\begin{array}{r}\text { Política } \\
\text { translocal }\end{array}$ \\
\hline $\begin{array}{l}\text { 1. Casa Retruco Solidaridad con } \\
\text { Argentina }\end{array}$ & Cívica & 1983 & 50 & Sí & No & Sí \\
\hline $\begin{array}{l}\text { 2. Red Solidaria Argentina en } \\
\text { Barcelona }\end{array}$ & Social & 2003 & 4 & Sí & Sí & Sí \\
\hline $\begin{array}{l}\text { 3. Grupo de Apoyo a la Central de } \\
\text { Trabajadores Argentinos }\end{array}$ & Cívica & 2001 & 10 & Sí & No & Sí \\
\hline $\begin{array}{l}\text { 4. Asociacion de Uruguayos en } \\
\text { Cataluña }\end{array}$ & Social & 2003 & 400 & Sí & Sí & Sí \\
\hline 5. Casa de Uruguay & Cívica & 1978 & 75 & Sí & No & Sí \\
\hline 6. Casa Charrua Uruguay & Cívica & 1992 & 10 & Sí & Sí & Sí \\
\hline 7. Asociación Panamá Cataluña & Cultural & 1997 & 50 & No & No & No \\
\hline 8. Asociacion Cultural Alma Peruana & Cultural & 1991 & 16 & Sí & No & Sí \\
\hline 9. Federacion de Peruanos sin Fronteras & Cívica & 1998 & 150 & Sí & No & Sí \\
\hline 10. Centro Peruano de Barcelona & Cultural & 1963 & 40 & Sí & No & Sí \\
\hline $\begin{array}{l}\text { 11. Associació Catalana-Equatorian per } \\
\text { a la integració i el desenvolpament }\end{array}$ & Social & 2003 & 5000 & Sí & No & Sí \\
\hline $\begin{array}{l}\text { 12. Associació d'Equatorians a } \\
\text { Catalunya }\end{array}$ & Social & 1993 & 7200 & Sí & No & No \\
\hline $\begin{array}{l}\text { 13. Asociación Inmigrantes } \\
\text { Ecuatorianos Solidaridad y } \\
\text { Cooperación -Ecuador Llactacaru }\end{array}$ & Social & 2001 & 20 & Sí & No & Sí \\
\hline $\begin{array}{l}\text { 14. Associació de Treballadors i } \\
\text { Immigrants Marroquins a Catalunya }\end{array}$ & Social & 1993 & 600 & Sí & No & Sí \\
\hline $\begin{array}{l}\text { 15. Asociación de Amigos del Pueblo } \\
\text { Marroqui - Itran }\end{array}$ & $\begin{array}{l}\text { Com. } \\
\text { origen }\end{array}$ & 1998 & 20 & Sí & No & Sí \\
\hline $\begin{array}{l}\text { 16. Asociación Cultural Rhombe } \\
\text { Guinea Ecuatorial }\end{array}$ & Cultural & 1983 & 200 & Sí & No & Sí \\
\hline $\begin{array}{l}\text { 17. Associació de Treballadors } \\
\text { Pakistanesos a Catalunya }\end{array}$ & Social & 1990 & 300 & Sí & No & Sí \\
\hline $\begin{array}{l}\text { 18. Associació Cultural Idara Minhach } \\
\text { Al Quoran Pakistan }\end{array}$ & Cultural & 1996 & 870 & Sí & No & Sí \\
\hline $\begin{array}{l}\text { 19. Federació d'Associacions } \\
\text { Pakistanesos a Catalunya }\end{array}$ & Social & 2004 & 190 & Sí & Sí & No \\
\hline $\begin{array}{l}\text { 20. Associació Cultural Educativa i } \\
\text { Social Operativa Dones Pakistaneses } \\
\text { (ACESOP) }\end{array}$ & Social & 2005 & 100 & no & si & si \\
\hline $\begin{array}{l}\text { 21. Associació Catalana de Residents } \\
\text { Senegalesos }\end{array}$ & Social & 1988 & 99 & Sí & Sí & Sí \\
\hline 22. Associació Nigeriana de Catalunya & Social & 1980 & 320 & Sí & Sí & No \\
\hline $\begin{array}{l}\text { 23. Agrupació Cultural Adna Bassa } \\
\text { Camerún }\end{array}$ & Cultural & 1996 & 50 & No & No & No \\
\hline $\begin{array}{l}\text { 24. Agrupació Camerunes Residents a } \\
\text { Catalunya }\end{array}$ & Social & 1985 & 15 & Sí & No & No \\
\hline
\end{tabular}


Los participantes entrevistados consideraban que era necesario que las asociaciones a las que pertenecían se implicaran en la política de los países de origen y de destino, y vinculaban dicha creencia a un sentimiento de doble pertenencia, que no parecía desvanecerse con el tiempo. Este sentimiento de dualidad fue claramente expresado por casi la totalidad de los representantes de las asociaciones participantes. Sin embargo, algunos entrevistados destacaron más aspectos identitarios y culturales:

"Vemos lo que sucede aquí porque vivimos aquí... No somos ciegos, vivimos todas las injusticias aquí porque somos parte de la sociedad catalana y participamos de ella. Y si algo sucede aquí, pedimos ayuda allí. Pero también somos argentinos y tenemos fuertes vínculos con nuestro país. Sería una gran injusticia no participar políticamente en la realidad de nuestro país. Es necesario hacerlo, al menos en memoria de todas aquellas personas que murieron por la democratización de la Argentina. Esta es la razón principal por la que nos unimos a esta asociación" (Presidente de la asociación).

Algunos representantes de las asociaciones participantes concebían la doble pertenencia como un sentimiento coherente con su pasado colonial, al proceder de países que fueron parte del Imperio Español, como es el caso de Guinea Ecuatorial:

"Yo soy un guineana aunque muchas veces, en broma, digo en mi lugar de trabajo que yo soy una mujer española exótica. Me siento guineana pero soy ciudadana española. Muchos de mis compatriotas en Guinea se sienten españoles. Mi madre habla perfectamente el español" (Miembro fundador de la asociación).

Los representantes de ciertas asociaciones manifestaron que la implicación en actividades políticamente transnacionales estaba relacionada con cuestiones socio-culturales, religiosas o ideológicas del país de origen. Factores tales como la presencia de un régimen opresor o no democrático en el país de origen, la falta de derechos políticos básicos en el país de destino (por ejemplo, la ausencia del derecho a voto) o el hecho de que en las asociaciones se encuentren dirigentes inmigrantes que llegaron con una fuerte identificación ideológica (anarquista, comunista, etc.) podría ayudar a comprender por qué ciertas asociaciones se involucran políticamente en el campo transnacional:

"Yo vengo de Marruecos, pero no pertenezco a ninguna comunidad política. Es más una pertenencia cultural y religiosa: pertenezco a la cultura árabe, pero yo no pertenezco a la comunidad política marroquí. Esta comunidad no permite 
mi presencia y yo tampoco quiero pertenecer a ella. Aunque esta es sólo mi visión. Me cuesta un poco hablar en nombre de todos los miembros de nuestra asociación, porque supongo que somos algo distintos. Aquí, en España o en Cataluña yo pertenezco a nuestra asociación. De momento, no estoy dispuesto a pertenecer a cualquier otra comunidad política" (Presidente de la asociación).

Sólo seis de las asociaciones participantes en el estudio se implicaban con regularidad en las elecciones de sus países de origen y tres asociaciones participaban cada año en diversos proyectos cívicos trasfronterizos. En general, se observa que el activismo político transnacional de las asociaciones de inmigrantes en Barcelona es de baja intensidad, con actividades y prácticas políticas que frecuentemente son poco institucionalizadas o de naturaleza ocasional.

Las asociaciones que se definían como "sociales" participaban en mayor medida en el transnacionalismo político, principalmente en la política de inmigración y la política del país de origen. La mayoría de estas asociaciones eran relativamente jóvenes (fueron fundadas después de 1990) y comparativamente tenían una mejor financiación (principalmente de fondos del gobierno), su agenda de acción era de alcance nacional, centrándose en varios proyectos en el país de origen en áreas como la educación y la salud.

Las entidades "cívicas" tendían a participar también en la constitución de proyectos políticos en su país de origen, generalmente a escala nacional. Con su vinculación política trasfronteriza, las asociaciones "culturales" trataban principalmente de preservar y difundir su cultura de origen en el país de destino. El único caso de asociación que se definía en términos de la comunidad de origen se dedicaba, principalmente, a proyectos de desarrollo comunitario local.

Como se observa en la Tabla 2, los resultados de este estudio refuerzan empíricamente el supuesto teórico de que las asociaciones de inmigrantes participan en grado diverso en los procesos de simultaneidad que los vincula políticamente a ambos lados de la frontera. A pesar de que la mayoría de asociaciones entrevistadas (91 por ciento) desarrollaron ciertas formas de implicación política transnacional (principalmente en la política del país de origen y la política trans-local), sólo una minoría realizaba estas actividades de forma regular. 
Tabla 2: Transnacionalismo político de las asociaciones de inmigrantes en Barcelona: formas, alcance y frecuencia

\begin{tabular}{|c|c|c|c|}
\hline Variable & Valores & Frecuencia & $\%$ \\
\hline \multirow{3}{*}{$\begin{array}{l}\text { Participación en } \\
\text { transnacionalismo político } \\
\text { Total }\end{array}$} & Sí & 22 & 91.7 \\
\hline & No & 2 & 8.3 \\
\hline & & 24 & 100.0 \\
\hline \multirow{4}{*}{$\begin{array}{l}\text { Formas de } \\
\text { transnacionalismo } \\
\text { político }{ }^{1} \\
\text { Total } \\
\end{array}$} & Políticas de inmigración & 6 & 25.0 \\
\hline & Políticas del país de origen & 21 & 87.5 \\
\hline & Políticas trans-locales & 18 & 75.0 \\
\hline & & 24 & 100.0 \\
\hline \multirow{3}{*}{$\begin{array}{l}\text { Alcance de los proyectos } \\
\text { cívicos en el país de } \\
\text { origen }^{2}\end{array}$} & Local & 1 & 4.5 \\
\hline & Regional & 1 & 4.5 \\
\hline & Nacional & 13 & 59.1 \\
\hline \multirow{5}{*}{$\begin{array}{l}\text { Foco de actividad en país } \\
\text { de origen }{ }^{3}\end{array}$} & Educación & 10 & 45.5 \\
\hline & Salud & 9 & 40.9 \\
\hline & Personas mayores y niños & 5 & 22.7 \\
\hline & Derechos Humanos & 2 & 9.1 \\
\hline & Desarrollo local & 6 & 27.3 \\
\hline \multirow{4}{*}{$\begin{array}{l}\text { Frecuencia de implicación } \\
\text { en elecciones nacionales } \\
\text { en el país de origen } \\
\text { Total }\end{array}$} & Nunca & 15 & 62.5 \\
\hline & Ocasionalmente & 3 & 12.5 \\
\hline & Cada elección nacional & 6 & 25.0 \\
\hline & & 24 & 100.0 \\
\hline \multirow{3}{*}{$\begin{array}{l}\text { Frecuencia de implicación } \\
\text { en proyectos cívicos en el } \\
\text { país de origen }\end{array}$} & Nunca & 8 & 33.3 \\
\hline & Ocasionalmente & 13 & 54.1 \\
\hline & Anualmente & 3 & 12.5 \\
\hline Total & & 24 & 100.0 \\
\hline
\end{tabular}

Fuente: elaboración propia. Los porcentajes no suman 100 ya que las asociaciones de inmigrantes pueden implicarse en diversas formas de transnacionalismo político. 2. Los porcentajes no suman 100 ya que las asociaciones de inmigrantes pueden implicarse en proyectos a diversos niveles o pueden no implicarse en proyectos nacionales. 3. Los porcentajes no suman 100 ya que las asociaciones de inmigrantes pueden implicarse en diversos proyectos en el país de origen.

\section{El PAPEL DEL CONTEXTO Y LAS CARACTERÍSTICAS ORGANIZATIVAS EN EL DESARROLLO DEL TRANSNACIONALISMO POLÍTICO}

Este estudio trata de explorar el papel que juegan determinados factores contextuales (la estructura de oportunidades políticas en el país de origen y el nivel de desarrollo socio-económico del país de origen), así como la relevancia de ciertas características organizativas (el tipo de asociación de inmigrantes, las redes sociales, el año de fundación o las fuentes de financiación) en la constitución de vínculos políticos a través de las fronteras.

El análisis que se presenta a continuación tiene un objetivo puramente heurístico y en ningún caso las relaciones observadas entre las variables 
pueden considerarse como causales, pues las inferencias que se han extraído proceden de una muestra pequeña. Sin embargo, las relaciones de asociación que se han hallado podrían alentar futuras investigaciones en el campo de la dimensión política del transnacionalismo migrante.

El efecto de la estructura de oportunidades políticas en el país de origen y del nivel socio-económico de la comunidad de origen han sido relativamente poco estudiados en el ámbito europeo (Smith 2003; Portes et al., 2007; Margheritis, 2007).

La presencia de situaciones políticas inestables o de transición en el país de origen puede generar una mayor implicación de los migrantes en la política de su país de origen (Portes et al., 2007). Las alianzas políticas inestables típicas de sistemas políticos pluralistas, la falta de competencia en los países menos democráticas, los conflictos entre las elites o derechos políticos restringidos pueden también motivar a los colectivos con bajos recursos (como los migrantes económicos) a asumir los posibles riesgos que conlleva la acción colectiva, y/o incentivar a las élites excluidas (como las diásporas) que adopten el papel de "la voz del pueblo" (Tarrow, 2004; Koopmans, 1999).

El activismo y las acciones transnacionales realizadas por las asociaciones de inmigrantes suelen variar entre los grupos de diferente origen étnico-cultural, en parte, debido a las diferentes políticas llevadas a cabo por los gobiernos de origen (Portes et al., 2007). Las políticas gubernamentales para los ciudadanos residentes en el extranjero tienden a desempeñar un papel importante en el campo social transnacional, especialmente si están diseñadas con objetivos que van más allá de apelaciones simbólicas y proporcionan ayuda real para los emigrantes (Smith, 2003).

Algunas iniciativas como las remesas o diferentes proyectos filantrópicos y cívicos llevados a cabo por parte de la población inmigrada en los países de origen pobres podrían tener un potencial contra-hegemónico frente a los procesos contemporáneos del capitalismo neoliberal global (Portes et al., 2007; Sandell et al., 2007; Itzigsohn y Saucedo 2002; Portes et al., 2003).

Mientras que el sistema económico neoliberal parece conducirnos a un aumento de las desigualdades entre países y en el interior de estos, las asociaciones de inmigrantes podrían aliviar tal situación mediante diferentes tipos de acción transfronteriza. Sin embargo, no es tan evidente que aquellas personas que han abandonado sus países de origen por causas relacionadas con la pobreza, la violencia o la falta de recursos y oportunidades, traten de cambiar tales condiciones mediante el uso de los recursos adquiridos en el extranjero (Portes et al., 2007). 
Los resultados del análisis bivariado muestran una relación de asociación entre las entidades que se dedican a la política del país de origen y el nivel de estructura de oportunidades políticas en el país de origen. Los datos parecen apuntar que una mayor estructura de oportunidades políticas en el país de origen está asociada a un mayor nivel de transnacionalismo político centrado en la política del país de origen y la política trans-local (H2) (Tabla 3).

Tabla 3: Transnacionalismo político por nivel de estructura de oportunidades políticas en el país de origen

\begin{tabular}{|c|c|c|c|c|c|}
\hline & \multicolumn{3}{|c|}{$\begin{array}{c}\text { Estructura de } \\
\text { oportunidades políticas }\end{array}$} & \multirow{2}{*}{$\begin{array}{l}\text { Coeficiente } \\
\text { de Pearson }\end{array}$} & \multirow{2}{*}{$\begin{array}{r}\text { V de } \\
\text { Cramer }\end{array}$} \\
\hline & Baja & Media & Alta & & \\
\hline Política del país de origen & $62.5 \%$ & $100 \%$ & $100 \%$ & $6.86^{* *}$ & $0.54 * *$ \\
\hline Política trans-local & $50 \%$ & $77.8 \%$ & $100 \%$ & $5.04 *$ & $0.46^{*}$ \\
\hline Política de inmigración & - & - & - & n.s & n.s \\
\hline
\end{tabular}

Se observa a la vez una posible asociación entre las entidades que se dedican a la política trans-local y el nivel de desarrollo socio-económico de sus países de origen. Las asociaciones de inmigrantes cuyos miembros provienen de países con un bajo nivel de desarrollo socio-económico parecen ser más propensas a no participar en la política transnacional. Este resultado contraviene la hipótesis inicial planteada (Tabla 4).

Tabla 4: Transnacionalismo político por nivel de desarrollo económico en el país de origen

\begin{tabular}{lrrrrr}
\hline & $\begin{array}{c}\text { Nivel de desarrollo } \\
\text { económico }\end{array}$ & $\begin{array}{r}\text { Coeficiente } \\
\text { de Pearson }\end{array}$ & $\begin{array}{r}\text { V de } \\
\text { Cramer }\end{array}$ \\
\hline Política del país de origen & - & - & - & n.s & n.s \\
Política trans-local & $25 \%$ & $84.6 \%$ & $85.7 \%$ & $6,4^{* *}$ & $0.51^{* *}$ \\
Política de inmigración & - & - & - & n.s & n.s \\
\hline $\begin{array}{l}\text { Nota: ** Significativo al nivel de } 95 \%, * \text { Significativo al nivel de } 90 \%, \text { n.s }=\text { no significativo. } \\
\text { Fuente: elaboración propia. }\end{array}$ & & & & &
\end{tabular}

En suma, el análisis exploratorio realizado apunta a que las dos variables contextuales estudiadas podrían estar asociadas a la participación de las asociaciones de inmigrantes en el transnacionalismo político. La 
relación de asociación parece más palmaria cuando ambos factores toman valores bajos. En otras palabras, las asociaciones de inmigrantes, cuyos miembros provienen de países pobres y con bajos niveles de estructura de oportunidades políticas, son menos propensas a participar en el transnacionalismo político. Por tanto, ambos factores, y en particular el nivel de estructura de oportunidades políticas en el país de origen, podrían ser condiciones necesarias (aunque no suficientes) para la participación política transnacional.

Algunos estudios revelan que las entidades cívicas suelen ser el tipo predominante de asociación de inmigrantes que mas participa en actividades transnacionales. El nivel y las fuentes de financiación suelen variar en función del tipo de entidad, hecho que puede afectar el nivel de implicación transnacional (Portes et al., 2007). Además, el capital social puede reducir los costes de la migración que perciben los potenciales migrantes internacionales, de tal forma que puede hacer o no factible el proyecto migratorio (Massey et al., 1993; Bashi, 2007; Piore, 1979). En el mismo sentido, cuanto mayor y más espacialmente diversificadas sean las redes, mayores deberían ser las posibilidades de que los migrantes se involucren en iniciativas políticas a través de las fronteras estatales (Guarnizo et al., 2003:. 1218-1224).

La exploración de factores organizacionales como el tipo de asociación, las fuentes de financiación, el número de miembros o el año de fundación señalan las fuentes de financiación como una variable posiblemente asociada al nivel de implicación de las asociaciones de inmigrantes en el transnacionalismo político. Aquellas asociaciones que reciben fondos o subvenciones del gobierno suelen involucrarse más en el transnacionalismo político. En concreto, los resultados indican que aquellas entidades que declaran recibir fondos del gobierno tienden a participar particularmente en la política del país de origen. Los límites de la muestra y la poca variación de los datos no son suficientes para calibrar el efecto del resto de características organizativas, por lo que nuevas investigaciones deberán abordar tales aspectos (Tabla 5).

Los datos muestran que la presencia de redes sociales más amplias suele aumentar la implicación en actividades y prácticas políticas transnacionales de las asociaciones entrevistadas. Sin embargo, características como el alcance o la diversificación espacial de las redes sociales no parecen estar asociadas a la participación política a través de las fronteras. 
Tabla 5: Transnacionalismo político por financiación gubernamental

\begin{tabular}{|c|c|c|c|c|}
\hline & \multicolumn{2}{|c|}{$\begin{array}{l}\text { Financiación } \\
\text { gubernamental }\end{array}$} & \multirow{2}{*}{$\begin{array}{l}\text { Coeficiente } \\
\text { de Pearson }\end{array}$} & \multirow{2}{*}{$\begin{array}{r}\mathrm{V} \text { de } \\
\text { Cramer } \\
\end{array}$} \\
\hline & No & Sí & & \\
\hline Política del país de origen & $57.1 \%$ & $100 \%$ & $8327 * *$ & $0.589 * *$ \\
\hline Política trans-local & - & - & n.s & n.s \\
\hline Política de inmigración & $0 \%$ & $35.5 \%$ & $329 *$ & $0.37 *$ \\
\hline
\end{tabular}

Este hallazgo es coherente con el conocimiento que disponemos acerca de cómo operan las redes sociales en la sociedad de la información, pues la orientación espacial de las redes sociales no suele ser tan relevante como la fluidez de la comunicación en el tiempo y el espacio, debido al impacto de las nuevas tecnologías de comunicación e información (TIC) (Castells, 1996; Castells, 2001; Cairncross 2001; Vancea y Olivera, 2013). Sin duda, una interesante línea de investigación futura es entender los mecanismos por los que las TIC pueden facilitar la participación de los inmigrantes en el activismo transnacional (Tabla 6).

Tabla 6: Exploración de factores determinantes meso y macro del transnacionalismo político. Regresión OLS

\begin{tabular}{lr}
\hline & Coeficientes \\
\hline Extensión de las redes sociales de la asociación & $0.048^{*}$ \\
Estructura de oportunidades políticas en el país de & $(0.024)$ \\
origen & $0.451^{* *}$ \\
Nivel de desarrollo económico en el país de origen & $(0.175)$ \\
& 0.069 n.s. \\
Constant & $(0.213)$ \\
F & 0.32 \\
R-squared & $(0.515)$ \\
Adjusted R-squared & $=0,011)$ \\
S.E.E. & $0.42 * *$ \\
N & $0.33 * *$ \\
Nota: $* *$ Significativo al nivel de $95 \%{ }^{*}$ * Significativo al nivel de $90 \%, \mathrm{n} . \mathrm{s}=$ no significativo.
\end{tabular}

Fuente: elaboración propia. 


\section{Conclusiones}

Este artículo ha mostrado que las asociaciones de inmigrantes en Barcelona se involucran en activismo político transnacional. Por consiguiente, el estudio presenta evidencia empírica que atestigua la existencia de un campo transnacional relativamente estable y significativo de acción política que conecta a las asociaciones inmigrantes con sus sistemas políticos de origen. Sin embargo, el ha mostrado también que la implicación política transnacional no sigue un patrón homogéneo y generalizado, presenta un nivel relativamente bajo de la regularidad y se basa, por lo general, en acciones que tienen un alcance $u$ objetivo nacional.

Los datos analizados en esta investigación han revelado la importancia que debería tener en los estudios sobre el transnacionalismo político de los inmigrantes hacer hincapié en el papel del contexto de origen y que no es posible ofrecer una explicación completa de transnacionalismo político inmigrante sin tener en cuenta esta dimensión. En concreto, la estructura de oportunidades políticas y el nivel de desarrollo socio-económico del país o comunidad de origen parecen ser unos indicadores claves para una comprensión cabal del fenómeno estudiado. La información recogida para esta investigación apuneta a que una mayor estructura de oportunidades políticas en el país de origen está asociada a un mayor nivel de transnacionalismo político, mientras que un menor nivel de desarrollo socio-económico del país o comunidad de origen conllevaría un menor nivel de implicación política transnacional.

Al nivel de análisis meso los datos sugieren que dos dimensiones, las redes sociales y las fuentes de financiación (fondos públicos), son elementos cardinales para comprender la variación en la participación política transnacional de las asociaciones de inmigrantes. Estar bien comunicado (y financiado) en el país de destino parece aumentar la probabilidad de que las asociaciones de inmigrantes lleven a cabo acciones políticas transnacionales.

El artículo ha mostrado que aplicar conceptos ampliamente utilizados en otros campos de estudio como el de la participación política o el de movimientos sociales, puede constituir una estrategia provechosa al tratar explicar la participación política transnacional de los inmigrantes. Los resultados de esta investigación muestran que la futura investigación en esta área de estudio deberá abordar el problema de la construcción de un modelo explicativo más integral que los manejados hasta el momento. 
En concreto, el estudio de las prácticas políticas transnacionales de 24 asociaciones de inmigrantes en Barcelona nos muestra el reto que supondrá en los próximos años el bastimento de un modelo explicativo que integre los tres niveles de análisis (individual, meso y macro) y que, a su vez, sea sensible a los contextos de salida y de destino. El estudio ha ahondado, principalmente, en los niveles meso y macro. La decisión de entrevistar a los líderes de las asociaciones no puede ser tomada como representativa de lo que ocurre a nivel individual en los colectivos de inmigrantes representados en el estudio.

Otros aspectos importantes que se deberían tomar en cuenta en futuros estudios son la influencia de los procesos macro políticos y macro económicos en la forma, intensidad y contenido de las prácticas políticas transnacionales, la dimensión e importancia del fenómeno estatal en los procesos de configuración del transnacionalismo político. Este estudio aporta información relevante para cartografiar la escala micro-social del transnacionalismo político, pero no pretendía indagar en la tensión permanente que existe entre distintas formas políticas estructurales como estados nacionales, bloques inter o transnacionales. Por su centralidad en el debate académico, tales cuestiones deberán abordarse en nuevas investigaciones. Finalmente, los resultados de este estudio también muestran la necesidad de ahondar en el escrutinio los efectos de esta forma de participación política en las instituciones y los sistemas políticos de los países de origen. Sólo así estaremos en situación de poder evaluar la naturaleza crítica o contra-hegemónica del transnacionalismo inmigrante.

\section{REFERENCIAS BIBLIOGRÁFICAS}

Basch, Linda, Glick Schiller, Nina y Szanton Blanc, Christina, 1994, Nations Unbound: Transnational Projects, Postcolonial Predicaments, and Deterritorialized Nation-States, PA: Gordon and Breach, Langhorne.

Bashi, Vilna, 2007, Survival of the Knitted. Immigrants Social Networks in a Stratified World, Stanford University Press, Stanford.

Bauböck, Rainer, 2003, "Towards a Political Economy of Migrant Transnationalism", in International Migration Review, vol. 37, núm. 3.

CAIRNCROSS, Frances, 2001, The death of distance: how the communications revolution is changing our lives, Harvard Business School Press, Boston.

Castells, Manuel, 2001, The Internet Galaxy, Oxford University Press, Oxford.

Castells, Manuel, 1996, The Information Age, vol. 1: The Rise of the Network Society, Blackwell, Oxford. 
Cohen, Robin, 2008, Global Diasporas: An Introduction, UCL Press, London.

Davis, Mike, 2006, "Planet of slums", in New Perspectives Quarterly, vol. 23, núm. 2.

Curtin, Philip D., 1984, Cross-cultural trade in world history, Cambridge University Press, Cambridge.

Gold, Steven J., 2002, The Israeli Diaspora, Routledge, New York.

Guarnizo, Luis Eduardo, Portes, Alejandro y Haller, William, 2003, “Assimilation and Transnationalism: Determinants of Transnational Political Action among Contemporary Migrants", in American Journal of Sociology, vol. 108, núm. 6.

Howe, Irving, 1976, World of Our Fathers, Harcourt Brace y Jovanovich, New York.

Itzigsohn, José, 2000, "Immigration and the Boundaries of Citizenship: the Institutions of Immigrants' Political Transnationalism", in International Migration Review, vol. 34, núm. 4.

Itzigsohn, José, y Giorguli Saucedo Silvia, 2002, "Immigrant Incorporation and Sociocultural Transnationalism", in International Migration Review, vol. 36, núm. 3.

Itzigsohn, José, Dore, Carlos, Hernández, Esther, y Vásquez, Obed, 1999, "Mapping Dominican Transnationalism: Narrow and Broad Transnational Practices", in Ethnic and Racial Studies, vol. 22, núm. 2.

King, Gary, Keohane, Robert y Verba, Sidney, 1994, Designing Social Inquiry, in Scientific Inference in Qualitative Research, Princeton University Press, Princeton.

Koopmans, Ruud, 1999, "Political. Opportunity. Structure. Some Splitting to Balance the Lumping", in Sociological Forum, vol. 14, núm. 1.

Koopmans, Ruud, Statham, Paul, Giugni, Marco y Passy, Florence, 2005, Contested Citizenship: Immigration and Cultural Diversity in Europe, University of Minnesota Press, Minneapolis.

Kymlicka, Will, 2003a, "Nationalism, Transnationalism and Postnationalism", in Dworkin, Ronald (coord.), From Liberal Values to Democratic Transition, Central European Press, Budapest.

Kymlicka, Will, 2003b, "New Forms of Citizenship", in Thomas, J. Courchene, T.J. y Savoie, Donald (coord.), The Art of State: Governance in a World without Frontiers, The Institute for Research on Public Policy, Montreal.

Kurien, Prema, 2001, "Religion. Ethnicity and Politics: Hindu and Muslim Indian Immigrants in the United States", in Ethnic and Racial Studies, vol, 24, núm. 2.

Landolt, Patricia, 2008, "The Transnational Geographies of Immigrant Politics: Insights from a Comparative Study of Migrant Grassroots Organizing", in The Sociological Quarterly, vol. 49, núm. 1. 
Levitt, Peggy y de la Dehesa, Rafael, 2003, “Transnational Migration and a Redefinition of the State: Variations and Explanations", in Ethnic and Racial Studies, vol. 26, núm. 4.

Levitt, Peggy y Glick Schiller, Nina, 2004, "Conceptualising Simultaneity: A Transnational Social Field Perspective on Society", in International Migration Review, vol. 38, núm. 3.

Luque Brazán, José Carlos, 2007, “Asociaciones políticas de inmigrantes peruanos y la "Lima Chica" en Santiago de Chile", in Migraciones Internacionales, vol. 4, núm. 2.

Margheritis, Ana, 2007, "State-led transnationalism and migration: reaching out to the Argentine community in Spain”, in Global Networks, vol. 7, núm. 1.

Martinello, Marco y Lafleur, Jean-Michel, 2008, "Towards a transatlantic dialogue in the study of immigrant political transnationalism", in Ethnic and Racial Studies, vol. 31, núm. 4.

Massey, Douglas S., Arango, Joaquin, Graeme, Hugo, Kouaouci, Ali, Pelegrino, Adela y J. Taylor, Edward, 1993, “Theories of International Migration: A Review and Appraisal", in Population and Development Review, vol. 19, núm. 3.

Menjivar, Cecilia, 2000, Fragmented Ties: Salvadoran Immigrant Networks in America, University of California Press, Berkeley y Los Angeles.

Milanovic, Branko, 2013, "Global Income Inequality in Numbers: in History and Now”, in Global Policy, vol. 4, núm. 2.

Moctezuma, Miguel, 2004, "Migración y formas organizativas en los Estados Unidos: los clubes y federaciones de migrantes mexicanos en California”, en G. Lanly y B. Venezuela (coord.), Clubes de Migrantes Oriundos Mexicanos en los Estados Unidos: la política transnacional de la nueva sociedad migrantes, Universidad de Guadalajara, Guadalajara.

Moctezuma, Miguel, 2008, "Transnacionalidad y transnacionalismo", en Papeles de Población, núm. 57.

Montero, José Ramón, Font, Joan y Torcal, Mariano, 2006, Ciudadanos, asociaciones y participación en España, Centro de Investigaciones Sociológicas, Madrid.

Morales, Laura y Jorba, Laia, 2010, "Transnational links and practices of migrants' organizations in Spain”, in R. Bauböck y T. Fais (coord.), Diaspora and Transnationalism, Amsterdam University Press, Amsterdam.

Noiriel, Gerard, 1995, "Russians and Armenians in France", in Cohen, Robin (comp.), The Cambridge Survey of World Migration, Cambridge University Press, Cambridge.

Ong, Aihwa y Nonini, Donald (coord.), 1997, Ungrounded empires: The cultural politics of modern Chinese transnationalism, Routledge, New York.

Ong, Aihwa, 1999, Flexible Citizenship: The Cultural Logic of Transnationality, Duke University Press, Durham, NC. 
Østergaard-Nielsen, E.K. (coord.), 2003a, International migration and sending countries: perceptions, policies and transnational relations, Palgrave Macmillan, Basingstoke.

Østergaard-Nielsen, Eva, 2003b, “The Politics of Migrants' Transnational Political Practices", in International Migration Review, vol. 37, núm. 3.

Østergaard-Nielsen, Eva, 2001, "Transnational political practices and the receiving state: Turks and Kurds in Germany and the Netherlands", in Global Networks, vol. 1, núm. 3.

Piore, Michael J., 1979, Birds of Passage: Migrant Labor in Industrial Societies, Cambridge University Press, Cambridge.

Portes, Alejandro, 1996, "Transnational Communities: Their Emergence and Significance in the Contemporary World System", in Korzeniewicz, Roberto Patricio y William, C. Smith, (coord.), Latin America in the World Economy, Greenwood Press, Westport, CT.

Portes, Alejandro, 1999, "Conclusion: Towards a new world - the origins and effects of transnational activities", in Ethnic and Racial Studies, vol. 22, núm. 2.

Portes, Alejandro, Escobar, Cristina y Walton Radford, Alexandria, 2007, "Immigrant Transnational Organizations and Development: A Comparative Study", in International Migration Review, vol. 41, núm 1.

Portes, Alejandro, Guarnizo, L. y Landolt, P., 2003, La globalización desde abajo: transnacionalismo immigrante y desarrollo. La experiencia de Estados Unidos y América Latina, Facultad Latinoamericana de Ciencias Sociales, FLACSO-México, México.

Portes, Alejandro, Guarnizo, Luis E. y Landolt, Patricia, 1999, "The study of transnationalism: pitfalls and promise of an emergent research field", in Ethnic and Racial Studies, vol. 22, núm. 2.

Rischin, Moses, 1962, The Promised City: New York Jews 1870-1914, Harvard University Press, Cambridge.

Solé, Carlota, 1995, "Portugal and Spain: from Exporters to Importers of Labour", en Cohen, Robin (comp.), The Cambridge Survey of World Migration, Imprenta de la Universidad de Cambridge, Cambridge.

Sandell, Richard, 2007, ¿Saltaron o les empujaron? El aumento de la inmigración subsahariana, Real Instituto Elcano de Estudios Internacionales y Estratégicos, Área Demografía y Población, working paper, 133/2007.

Smith, R.C., 2003, "Migrant membership as an instituted process: transnationalization, the state and the extra-territorial conduct of Mexican politics", in International Migration Review, vol. 37, núm. 2.

Smith, Michael Peter y Guarnizo, Luis (coord.), 1998, Transnational from Below: Comparative Urban and Community Research, Transaction Publishers, New Brunswick and London. 
Tarrow, Sidney, 2004, El poder en movimiento. Los movimientos sociales. La acción colectiva y la política, Alianza Editorial, Madrid.

Vancea, Mihaela y Olivera, Nihil, 2013, "E-migrant Women in Catalonia: Mobile Phone Use and Maintenance of Family Relationship", in Gender, Technology and Development, vol. 17, núm. 2.

Vertovec, Steven, 2004, "Migrant Transnationalism and Modes of Transformation”, in International Migration Review, vol. 38, núm. 3.

Vertovec, Steven, 2009, Transnationalism, Routledge, New York.

Well, Patrick, 1991, La France et ses Etrangéres, Gallimard, París.

\section{Resumen Curricular de los autores}

\section{Mihaela Vancea}

Es Doctora en Ciencias Políticas y Sociales por la Universidad Pompeu Fabra (Barcelona, España). Acutalmente, trabaja como investigadora y profesora en el Núcleo Científico y Tecnológico de Ciencias Sociales y Humanidades de la Universidad de la Frontera, Chile y en el Departamento de Ciencias Políticas y Sociales de la Universidad Pompeu Fabra, España. Entre sus últimas publicaciones se encuentran (en coautoría): "Population Aging in the European Information Societies: Towards a Comprehensive Research Agenda in eHealth Innovations for Elderly" (2016), Aging and Disease, 7(5) o "Migrant Women and labour integration in Catalonia: the impact of new information and communication technologies" (2015), Revista de Estudios Sociales, 53.

Dirección electrónica: mihaela.vancea@ufrontera.cl; mihaela.vancea@ upf.edu

\section{Àlex Boso}

Es doctor por el Departamento de Sociología de la Universidad de Barcelona. Actualmente, es profesor e investigador del Núcleo Científico Tecnológico en Ciencias Sociales y Humanidades de la Universidad de la Frontera, Temuco, Chile. Ha sido profesor asociado del Departamento de Ciencias Políticas y Sociales de la Universidad Pomeu Fabra y de la Facultad de Salud y Acción Social de la Universidad de Vic. Ha colaborado como investigador y asistente técnico en el Centro de Investigación Sociotécnica del CIEMAT y en el programa Migration and Network Society del Internet Interdisciplinary Institute de la Universitat Oberta de Catalunya. Sus principales áreas de estudio son el análisis de las políticas públicas y 
las relaciones entre salud, medioambiente y migraciones. Entre sus últimas publicaciones se encuentra (en co-autoría):"Should irregular migrants have the right to healthcare? Lessons learnt from the Spanish case" (2016), Critical Social Policy, 36(2).

Dirección electrónica: alex.boso@ufrontera.cl

Artículo recibido el 22 de abril de 2016 y aprobado el 20 de mayo de 2017. 